\title{
AVALIAÇÃO ABERTA PELOS PARES NO ÂMBITO DA CIÊNCIA ABERTA: REVISÃO E REFLEXÃO
}

\begin{abstract}
Milton Shintaku
Doutor em Ciência da Informação pela Universidade de Brasília (UnB). Atua em pesquisas sobre tecnologia para gestão da informação no Instituto Brasileiro de Informação em Ciência e Tecnologia (lbict). milton.shintaku@gmail.com https://orcid.org/0000-0002-6476-4953
\end{abstract}

Rui Seabra Ferreira Jr. Doutor em Doenças Tropicais pela Faculdade de Medicina da Universidade Estadual Paulista Júlio de Mesquita Filho (UNESP). Pesquisador Adjunto do Centro de Estudos de Venenos e Animais Peçonhentos. na Universidade Estadual Paulista Júlio de Mesquita Filho (UNESP). ruicevap@gmail.com https://orcid.org/0000-0001-6952-0512
Ronnie Fagundes de Brito

Doutor em Engenharia e Gestão do Conhecimento pela Universidade Federal de Santa Catarina (UFSC). Atua em pesquisas sobre Mídia e Conhecimento na Educação e Acessibilidade. ronniebrito@ibict.br https://orcid.org/0000-0002-3979-603X

Benedito Barraviera

Doutor em Medicina Interna pela Universidade Estadual Paulista Júlio de Mesquita Filho (UNESP). Professor Titular de Infectologia pela Faculdade de Medicina de

Botucatu (UNESP-FMB) bbviera@gmail.com http://orcid.org/0000-0002-9855-5594

\section{RESUMO}

As Ciências mudam e evoluem constantemente seus processos. Dentre eles, destaca-se o movimento de abertura, o qual se distingue em face da pressão maciça da Tecnologia da Informação e Comunicação (TIC). A Ciência Aberta tem se mostrado a mais impactante por englobar várias iniciativas, sendo a Avaliação Aberta pelos Pares o maior desafio. O presente estudo teve por objetivos levantar e analisar a literatura disponível sobre a Avaliação Aberta pelos Pares a fim de identificar conceitos e tendências e apoiar os editores na discussão sobre essa nova modalidade proposta. A avaliação aberta pelos pares ainda se apresenta como um desafio para todos os envolvidos na editoração científica. Apesar das vantagens teóricas, a avaliação duplo-cega reina absoluta, ainda se questionando se tanto o avaliado quanto 0 avaliador devem se conhecer. A abertura das ciências é um processo aparentemente irreversível, embora esteja enfrentando muita resistência pelos produtores do conhecimento científico. A implantação da avaliação aberta permitirá, em futuro breve, o acompanhamento e a avaliação deste processo, o que culminará com procedimentos transparentes e fornecerá novos subsídios tanto para os editores quanto para os avaliadores/avaliados.

Palavras-chave: Ciência Aberta. Fluxo editorial. Avaliação pelos pares. Avaliação aberta. Periódicos científicos.

\section{OPEN EVALUATION BY PEOPLE IN THE FRAMEWORK OF OPEN SCIENCE: REVIEW AND REFLECTION}

\begin{abstract}
Sciences constantly change e evolve their processes. Among them, the open access movement stands out, facing the pressure of Information and Communication Technology (ICT). Open Science has proved to be the most impactful as it encompasses several initiatives, with Open Peer Review being the biggest challenge. This paper aims to survey and analyze the available literature on Open Peer Review, in order to identify concepts and trends and support the editors in the discussion about this proposed new modality. Open Peer Assessment still presents itself as a challenge for scientific publishing. Despite the theoretical advantages, double-blind evaluation is still absolute, even when questioning whether both the appraisers and the appraiser should know each other. The Open Science movement is irreversible, although it is facing resistance by the producers of scientific knowledge. The implementation of Open Peer Review will allow the monitoring and evaluation of this process, which will culminate in transparent procedures and provide new subsidies for both editors and reviewer.
\end{abstract}

Keywords: Open Scienci. Publishing editors' cycle. Peer Review. Journals.

Recebido em: 01/04/2020

Aceito em: $31 / 07 / 2020$
Publicado em: 11/09/2020 


\section{INTRODUÇÃO}

No final do século $\mathrm{XX}$, alavancado pela evolução tecnológica da Internet e Web, surgiu o movimento dos arquivos abertos (Open Artchives), que impactou as ciências, principalmente pelo fomento à criação das bibliotecas digitais de teses e dissertações e pela sua interoperabilidade. Com isso, possibilitou o surgimento de agregadores, como a Networked Digital Library of Theses and Dissertations (NDLTD) ${ }^{1}$, que agrega teses e dissertações de todo o mundo, ou a Biblioteca Digital de Teses e Dissertações (BDTD) ${ }^{2}$, no Brasil.

Quase que, paralelamente, por causa da chamada crise dos periódicos, surgiu o movimento de acesso aberto (Open Access), que defende a abertura dos resultados de pesquisas, principalmente pelos periódicos de acesso aberto, a denominada "via dourada", por (HARNAD, 2004). No Brasil, vale destacar a criação do Scientific Electronic Library Online (SciELO), que é a primeira iniciativa voltada para a disseminação, sem restrições, de artigos científicos publicados em revistas abertas, cujo modelo é exportado para vários países.

$\mathrm{Na}$ abertura das ciências, mas apresentando inovação, a criação do projeto ainda vigente ArXiv, em 1991, repercutiu por publicar os denominados preprints, da área de física e, depois, expandi-los para outras disciplinas, em iniciativa do laboratório da Universidade de Los Alamos, Estados Unidos.Como resultado, Ginsparg (2011), o seu criador, relata que o ArXiv está imerso num mundo em transformação, apoiado pela revolução tecnológica, com uma quantidade vasta de literatura científica, que ainda se mantém atual, apesar da idade.

Outra iniciativa de abertura das atividades científicas são os dados abertos (Open Data), que têm repercussão no governo, com os dados abertos governamentais e dados abertos de pesquisa. Eles fomentam a disseminação dos dados de pesquisa, respeitando as questões éticas e jurídicas em repositórios de dados, para que outros pesquisadores os reutilizem sem restrições, promovendo maior transparência nas pesquisas.

Assim, resumidamente, o Arquivo Aberto promoveu a disponibilização online das teses e dissertações, até então, restrita às bibliotecas depositárias; o acesso Aberto, por sua vez, viabilizou a disponibilização sem restrição aos artigos de pesquisa, por meio de

\footnotetext{
${ }^{1}$ Disponível em: http://www.ndltd.org/

${ }^{2}$ Disponível em: http://bdtd.ibict.br/vufind/
} 
revistas de acesso aberto ou em repositórios; o ArXiv deu acesso aos preprints, e, os Dados abertos deram acesso aos dados de pesquisa. Um fato em comum entre todos esses movimentos é que eles estão contextualizados, nas práticas científicas e, além disso, estão quebrando barreiras, em relação ao acesso aos dados e informações.

Entretanto, pode-se afirmar que o movimento mais impactante nos processos científicos é a Ciência Aberta, por não se restringir a um único aspecto da comunicação científica, como no caso dos outros movimentos, mas englobar várias iniciativas. Como relata Albagli, Clínio e Raychtock (2014), a Ciência Aberta é um guarda-chuva, que agrega práticas, significados e iniciativas voltadas a tornar a ciência mais democrática e participativa, por meio da valorização de todos que estão envolvidos em fazer ciência, mesmo aqueles que são considerados leigos.

A ideia de uma ciência mais aberta e democrática não é tão recente, tanto que Cole (1993) apresenta o cenário do final do século passado nas pesquisas americanas defendendo alterações em suas práticas, uma vez que as mudanças sociopolíticas encontram-se necessitadas de fazer mais com menos. David (1991) previu que a, então, Ciência Aberta seria uma revolução nas ciências, antes das mudanças ocorrerem. Do mesmo modo, David (1991) advoga que condutas da Ciência Aberta são mais adequadas ao momento em que ocorrem restrições de recursos, estado em que os cientistas estão mais preparados para operar em situações mais restritivas.

Mais recentemente, Milham e Klein (2019) defenderam que a Ciência Aberta (CA) fomenta a prática de compartilhamento de recurso, o que pode diminuir a discrepância entre os que possuem maior financiamento e quem recebe menos, visto que pesquisa de ponta é item dispendioso. Nesse mesmo caminho, Banks et al. (2019) salientam a necessidade de promover o compartilhamento, para a melhoria da qualidade de resultados de pesquisa. Os autores apelam aos editores que se engajem em práticas da CA, como o pré-registro e o compartilhamento de dados, assim como em estudos para verificação da eficácia das avaliações pelos pares.

Entretanto, um dos aspectos da CA que ainda provoca discussões é a Revisão Aberta pelo Pares (RAP) ou, em inglês, Open Peer Review (OPR). Como explica Silva (2019), semelhante impasse se deve pelo medo de represálias e possíveis críticas, as quais podem afetar a vida acadêmica, mesmo que resultem em pontos positivos de transparência. Esse quesito pode afetar a adesão dos editores ao uso desta modalidade 
de avaliação, mantendo a avaliação tradicional de dupla cega, mesmo que em comunidades pequenas de pesquisadores, tal tipologia de avaliação não funcione bem.

A própria definição de revisão aberta pelos pares não é clara. Ross-Hallauer (2017) relata mais de uma centena de definições para RAP, muitas das quais conflitantes. Essa indicação demonstra ainda a necessidade de discussão sobre o tema, de forma a buscar consensos, para criação de padrões aceitos pela comunidade.

No Brasil, há outro agravante, relacionado à pouca literatura sobre essa modalidade de avaliação. Em sites brasileiros, numa busca simples por termos como "avaliação aberta de artigos", "avaliação aberta pelos pares" ou "open Peer Review", no máximo encontram-se 114 documentos, os quais nem sempre tratam do assunto, mas apenas mencionam o termo, além de citarem artigos estrangeiros.

Nesse contexto, a presente análise tem por finalidade estudar o processo de avaliação aberta pelos pares, a fim de apresentar embasamento teórico voltado ao editor científico, que possibilite ou não a adesão a essa modalidade. Ademais, busca-se contribuir com a discussão sobre a CA, especificamente em relação ao RAP, com vistas a atender à pouca literatura acerca do tema, que pode ser importante nas publicações de Acesso Aberto.

\section{METODOLOGIA}

O estudo possui características exploratórias, na medida em que visa proporcionar maior familiaridade à Revisão Aberta pelos Pares (RAP), levantando seus conceitos e práticas alinhados à definição de Gil (2008). A pesquisa se ancora nas acepções de estudos exploratórios apresentados por Raupp e Beuren (2013), com algumas ressalvas, em que se defende a exploração de um assunto, como forma de reunir conhecimentos, incorporando novos, ainda não analisados, mesmo que, neste caso, não seja um objeto de estudo inédito.

Da mesma forma, a pesquisa caracteriza-se como, estritamente, qualitativa, na medida em que utiliza coleta de dados baseados em levantamentos bibliográficos e documentais, sem o uso de dados quantitativos ou técnicas estatísticas. Nesta pesquisa, a coleta de dados ocorre por meio de artigos, livros (bibliográficos) e páginas, portais e sites disponíveis na Web (documental), buscando por informações que atendam às definições e atividades relacionadas à avaliação aberta. 
A principal fonte de dados foi o Google Acadêmico, uma vez que ele abrange uma vasta coleção de periódicos e livros que, de acordo com Harzing (2019), ainda se mantêm como boa opção para levantamentos bibliográficos, tendo a vantagem de ser de acesso livre, se comparado com Web Of Science, Scopus, Cross Ref e outros. A oferta de um motor de busca, aliada à indexação das principais fontes acadêmicas, torna o Google Scholar uma boa opção para pesquisas bibliográficas, principalmente com o objetivo de levantar bibliografia sobre Ciência Aberta.

$\mathrm{Na}$ fase de coleta, a busca se deu, prioritariamente, em inglês, visto que a literatura técnico-científica se apresenta, sobretudo, nesse idioma, com argumento de busca centrado em "Open Peer Review". Com isso, o resultado de busca possibilitou recuperar os documentos completos para análise, buscando os conceitos e tendências relacionadas à Ciência Aberta, na medida em que o tema pode surgir em outros contextos.

Entretanto, como a avaliação é uma atividade intermediária ao fluxo editorial, pode ser que não seja totalmente explorada na literatura técnico-científica, manifestando certas restrições. Assim, o presente estudo se apresenta como inicial, não tendo a intenção de ser extenso ou abrangente, mas buscando contribuir com a discussão na área de editoração científica.

\section{RESULTADOS E DISCUSSÕES}

A questão da avaliação aberta não é algo novo, voltado exclusivamente à Ciência Aberta (CA), pois Rooyen et al. (1999) revelam que o fato de autores e avaliadores conhecerem as identidades de ambos pode não influenciar na qualidade dos pareceres, mas pode afetar a probabilidade de os avaliadores declinarem do convite para revisão. Esse ponto revela, entre outras questões, o receio que ainda cerca a avaliação aberta como mudança no processo de avaliação científica. Já Walsh et al. (2000) verificaram que, geralmente, os avaliadores afirmam não se importar com a avaliação aberta, mas aprovam mais trabalhos, sendo mais corteses, com melhor qualidade de parecer.

Falagas (2007), discutindo a abertura das ciências, já defende a mudança no processo de avaliação pelos pares, com o objetivo de torná-la mais transparente. Com isso, revela a preocupação das mudanças na ciência, principalmente em relação à ciência aberta e o seu impacto na comunicação científica. Cabe destacar que a mudança da avaliação cega, ou duplamente cega, para a aberta, com a publicação dos pareceres, 
é mais política que técnica, na medida em que, tecnologicamente, não há empecilhos para a adesão. Bravo (2019) destaca que a Revisão Aberta pelos Pares (RAP) ainda é vista com desconfiança, apesar dos benefícios, pois muitos revisores sentem-se desprotegidos quanto à anonimidade, revelando fortes problemáticas culturais existentes no processo de publicação.

Schmidt et al (2018) apresenta questões sobre a RAP, que merecem ser discutidas, sendo as principais:

- Não há uma única modalidade: ainda há questões em aberto, como: o parecer será publicado? o trabalho será rejeitado? ou o parecerista e os autores vão discutir o parecer? entre outras perguntas ainda sem consenso;

- Fomenta a confiança mútua, o respeito e a abertura às críticas: a avaliação torna-se um diálogo para melhoria do trabalho, durante o qual os leitores podem acompanhar as recomendações e os resultados;

- Proporciona qualidade construtiva e transparência com responsabilidade: a publicação dos pareceres garante a transparência e apoia a melhoria do trabalho, numa contribuição construtiva;

- Possibilita o reconhecimento aos revisores e torna as avaliações citáveis: Pareceres publicados dão visibilidade aos avaliadores, possibilitando que esses relatórios possam ser citados.

Já Ross-Hellauer e Görögh (2019), na intenção de facilitar a sua implementação, apresentam uma lista de conselhos para a adoção da RAP, em apoio à política de RAP:

- Definir as metas:

- Definir quais as metas a serem alcançadas pela RAP: A implementação do RAP requer conscientização dos objetivos a serem alcançados com essa nova modalidade de avaliação e quais os pontos querem melhorar no processo.

- Conhecer todas as possibilidades ofertadas pela RAP: A avaliação aberta não é uma modalidade única. Assim, pode-se responder às perguntas:

- Identificação dos autores e pareceristas:

- Autores e pareceristas vão ter suas identidades abertas, durante o processo de revisão?

- Publicação da avaliação:

- As avaliações serão publicadas? 
- Possibilidade da avaliação pela comunidade:

- A avaliação será à comunidade a contribuição para a revisão?

- Possibilidade de interação entre autor e avaliador:

- Haverá a possibilidade de interação entre os autores e revisores?

- Disponibilização do preprint:

- Na submissão, o manuscrito fica aberto como preprint?

- Comentários pós-publicação:

- Será aberta a possibilidade de os leitores comentarem os artigos publicados?

\section{- Plataformas abertas:}

- O processo de revisão será facilitado por outra instituição.?

- Decidir quais elementos quer implementar: Ao ter os objetivos a serem alcançados, criar estratégias para implementação, estudar modelos implementados e discutir e aprender com outras experiências.

\section{- Ouvir a comunidade}

- Atender às diferenças entre comunidades: Tenha consciência das diferenças entre as comunidades, em relação aos tipos de revisão. Algumas são mais tradicionais e outras mais abertas a mudanças.

- Considerar consulta aos leitores, autores e avaliadores: Considerar fazer um levantamento sobre o assunto, principalmente para revistas de associação ou que queiram se aproximar dos leitores.

- Envolver os stakeholders no processo: envolva o conselho editorial, revisores e todos aqueles que possam ter importância durante o processo de implementação.

- Planejar tecnologias e custos: implementação da revisão aberta pode impactar a tecnologia utilizada pela revista, assim como ter custos.

- Avaliar tecnologias: verificar se a tecnologia utilizada pela revista suporta a modalidade de RAP adotada;

- Avaliar custos: Verificar se a implantação do RAP terá custos, se será preciso desenvolver algo, ou mesmo adaptar processos. 
- Considerar opções alternativas para testar: caso queira testar, antes de implementar, mas tenha a consciência de que os resultados dos testes podem não ser os mais fidedignos, por isso, crie estratégias com mais de uma opção de teste.

\section{- Ter uma abordagem pragmática}

- Definir prioridades: mudanças podem ser difíceis, então priorize as mudanças, pois fazer tudo de uma vez pode apresentar desafios muito grandes.

- Testar pilotos: pode-se começar colocando algumas opções como opcionais para ver a aceitação, pois mudanças bruscas, na política da revista, podem trazer prejuízos.

\section{- Ter plano para divulgação dos conceitos}

- Repassar ideia à comunidade: após decidir o que implementar, deve repassar a todos. Assim, como estratégia, comece pelos colaboradores mais receptivos, da mesma forma que deve enfatizar as vantagens do RAP.

- Dar atenção à comunicação e terminologia: interpretações errôneas podem atrapalhar, assim, utilize meio para divulgar as novas práticas, além de escrever as políticas editoriais de forma fácil e simples.

\section{- Avaliar desempenho}

- Ter claramente o que vai analisar como desempenho: é preciso analisar a qualidade das avaliações e o índice de aceitação para avaliar o processo. Assim, deve-se ter uma clara ideia do que é sucesso na implantação do RAP. Algumas perguntas podem ajudar.Entre elas:

- A qualidade da revisão foi melhorada?

- Está mais difícil encontrar revisores?

- O tempo de revisão foi impactado?

- As revisões estão sendo consultados e reutilizados?

- Aceitar que mudanças levam tempo: a aceitação das mudanças pode não ser imediata, requerendo avaliações voltadas a ajustes. No entanto, os ganhos podem ser maiores e requererem persistência.

- Apresentar as avaliações para a comunidade: Após avaliação, manter os usuários a par dos resultados, da mesma forma que pensar em publicar os resultados como trabalho científico, podendo contar com 0 apoio de pesquisadores cm comunicação científica. 


\section{- Conselhos na implementação}

- Identidades abertas para autores e avaliadores: modalidade pela qual, tanto o autor, quanto o parecerista têm sua identidade partilhada, ou seja, o autor sabe quem está avaliando seu trabalho e o avaliador sabe o nome dos autores:

- Ter estratégias para obter um corpo de avaliadores que aceitam essa modalidade: ter uma comunicação clara com os pareceristas, apresentando as vantagens e desvantagens do processo, podendo flexibilizar tempo para avaliação.

- Estar alerta para interações conflituosas: ter estratégias para atuar com o receio de represálias por parte dos pareceristas, visto o medo de serem prejudicados, se rejeitarem trabalhos de pesquisadores renomados.

- Dar o crédito apropriado: proporcionar identificação completa dos autores e avaliadores, como o ORCID, a fim de aumentar a visibilidade da avaliação.

- Testar, primeiro, ou colocar a identidade aberta opcional: pode-se criar estratégias de adoção do RAP gradual, iniciando pela apresentação dos pareceristas na edição, até a abertura total da avaliação pelos pares.

- Publicar a avaliação junto a artigo aceito: em uma revisão aberta, o relatório de avaliação é publicado junto ao artigo, até para reuso, se for o caso, dando a devida autoria da avaliação.

- Encontrar melhor forma para publicação da avaliação:Não há modelo padrão, mas pode ser como documento adicional ou na apresentação do artigo. A melhor sugestão seria a publicação, como um documento adicional, com DOI diferente do artigo, para uso, mas poderia ser:

- publicar as revisões como anexo, procurando mecanismos para associar o relatório ao artigo, pelos metadados.

- publicar as revisões como parte do artigo, como um componente ou subartigo.

- publicar, como um artigo complementar ou comentário, com vinculações bidirecionais.

oEstar ciente dos desafios na publicação dos pareceres: a política editorial deve ser clara para evitar problemas, nos conteúdos dos pareceres deve-se evitar comentários depreciativos, entre outros. 
o Participação aberta, desde o preprint ao posprint: Em uma avaliação aberta mais ampla, o processo é totalmente transparente, com a possibilidade de participação de todos, desde a submissão, com a publicação do preprint, até a habilitação dos comentários, no posprint.

- Decidir quem pode comentar: deve-se decidir se as contribuições podem ou não ser anônimas, ouse somente pessoas registradas o podem fazer.

- Fomentar a participação da comunidade: a adesão à RAP nem sempre é boa, assim, requer o fomento à participação, podendo ser feito pela mediação da comunidade.

- Interação aberta: fomentar a possibilidade dos pareceristas e autores interagirem.

- Definir o fluxo de interação a ser implementado: se abrir a interação, deve-se definir o fluxo que essa interação pode ocorrer.

- Estar ciente da carga adicional de trabalho: Em alguns casos, a carga de trabalho do editor pode aumentar, mas, em outros, ela diminui, como nos casos de impasse, visto que os pareceristas e autores podem chegar a um consenso mais facilmente.

Barrett (2018), por sua vez, revela que a RAP pode apoiar o aprendizado a novos pesquisadores, na medida em que, ao publicar as avaliações, pareceristas em potencial podem aprender como fazê-lo. Esse ponto se alinha ao pensamento de que a RAP é uma ferramenta de aprendizado para outros avaliadores, visto que indícios revelam que pareceres abertos são melhor apresentados, com maior cortesia e colaboração para melhoria do trabalho.

Para Rabkina (2019), a RAP ainda não se consolidou, emergindo em vários formatos e embasado em vários conceitos, suportado pelas mudanças filosóficas e tecnológicas, sendo o caminho a ser adotado, futuramente. Além disso, as variações, ainda presentes, representam a flexibilidade das práticas e a consequente viabilidade do fenômeno emergente. Assim, para a autora, as variações apresentadas nas práticas relacionadas a RAP se devem à necessidade de adequação ao ambiente atual, em transição do fechado para o aberto. Acrescenta-se a isto o fato de que, no futuro, todas as avaliações serão abertas. 
Um dos pontos relacionados ao processo de avaliação é que o parecerista deve ser conhecedor do assunto do trabalho a ser avaliado. Assim, com a RAP, um canal de diálogo pode ser criado entre parecerista e autor, com vistas a melhorar o trabalho. Consequentemente, Lobo (2019) sugere que os avaliadores abertos podem ser convidados a ser coautores, mesmo que esse procedimento requeira um código de ética bem estruturado, na medida em que colaboram como texto, se aberto à discussão entre autores e avaliadores.

Polka, Ross-Hellauer e McDowell (2019) relatam que não há uma única prática na implementação da revisão aberta pelos pares, visto que podem ser adotadas formas diferenciadas, tais como:

- Revisão aberta pelos pares, como um opcional ou mandatório;

- Quando da decisão de publicar os relatórios de revisão, antes ou depois da revisão;

- O que é publicado como relatório de revisão, o texto completo com todos os comentários ou apenas uma compilação da revisão;

- O relatório de revisão é um adendo ao artigo ou tem vida própria, com direito a Digital Object Identifier (DOI).

Ford (2019), por seu lado, argumenta que a RAP deva ser implementado para fomentar a transparência nas atividades de publicação, deixando claras as políticas editoriais, explicitando as diretrizes, assim como as responsabilidades dos autores, pareceristas, editores e membros dos conselhos. $O$ autor ainda relata que a comunidade acadêmica deve assumir a tarefa de adotar a revisão aberta com disseminação, treinamentos e educação, visto que não é de interesse das editoras comerciais adotar essa prática, sem que ela venha acompanhada de lucro, por exemplo.

Como dito anteriormente, a tecnologia é aliada na abertura das revisões. Assim, Jana (2019) aponta a tecnologia Block Chain para essa prática, no intuito de garantir o reconhecimento dos revisores, remover vieses sexistas e outros, fomentando a transparência. Nesse sentido, o processo de revisão aberta pelos pares torna-se uma transação passível de registro seguro, em escala mundial, pois ofertará a veracidade dos registros.

O uso de novas tecnologias e iniciativas como o Researchgate pode ser útil nas publicações, mesmo na adoção da avaliação aberta, como relatam Alexander et al (2020). Nesse sentido, os mesmos autores mostram como a tecnologia tem apoiado 
as mudanças ocorridas na comunicação científica, desde os primeiros passos da abertura das ciências, mesmo que ainda não se tenha vislumbrado todas as possibilidades e potencialidades.

Klebel et al (2020), ao discutir a clareza das políticas de avaliação pelos pares, apresentam fortes indícios dos problemas com que os editores se deparam ao descreverem os processos da avaliação aberta, os quais, nem sempre, ficam claros. Esse ponto realça a necessidade de disseminar conceitos e práticas sobre a avaliação aberta, a fim de que toda a comunidade acadêmica possa participar da discussão sobre a adoção desta modalidade de revisão.

Barrett (2019), por sua vez, descreve a RAP como uma das mais significativas ações implementadas na Revista Proceeding of Royal Society $B^{3}$ : Biological Sciences (Proceeding B), mesmo com a oposição enfrentada. A Proceeding $B$ publica os relatórios de avaliação na forma de um arquivo complementar, sendo uma das mais respeitadas publicações na área de Biologia, com publicação ininterrupta na área desde 1905, mas com criação original em 1800, vinculada à Royal Society, com fator de impacto de 4.8 em 2017. No Brasil, a Journal of Venomous Animals and Toxins including Tropical Diseases (JVATiTD), a Memórias do Instituto Oswaldo Cruz (MIOC) e a Revista da Sociedade Brasileira de Medicina Tropical (RSBMT), três publicações da área de Medicina Tropical, responderam ao chamado da SciELO para adotarem premissas da Ciência Aberta, incluindo a avaliação aberta, como relata Barraviera et al. (2020). Nota-se, portanto, que a RAP é uma inovação a ser adotada, na medida em que revistas respeitadas no mundo a estão adotando.

Cabe destacar que a avaliação aberta é complexa e possui várias questões que ainda estão em discussão ou que não estão totalmente conceituadas. Do mesmo modo, ela possui vários pontos de customização, além de uma flexibilidade que possibilita, ao editor, adequá-la da forma que melhor atender a revista.

\section{CONSIDERAÇÕES FINAIS}

A discussão sobre a Revisão Aberta pelos Pares (RAP) é recente, no Brasil, com escassa literatura em português. Apesar de, teoricamente promissora, apresenta complexidades a resolver, tanto que Amaral e Príncipe (2018) indicam alguns desafios

\footnotetext{
${ }^{3}$ Disponível em: https://royalsocietypublishing.org/journal/rspb
} 
e reflexões que acometem a implementação desta nova modalidade, destacando-se os problemas acadêmicos que poderão advir, no futuro, entre autores e avaliadores.

A mudança na forma de avaliação, da fechada para a aberta, envolve alterações comportamentais, nos processos tradicionalmente estabelecidos e aceitos pela comunidade que trabalha nas publicações científicas. Isso pode acarretar nas desconfianças que acometem a adesão a este processo, requerendo ações proativas dos editores, de forma a se ter um ambiente favorável à sua implementação.

Entretanto, pode-se dizer que a necessidade de transparência nas atividades editoriais torna-se cada vez mais requerida, encontrando no RAP um pilar para a sua sustentação, visto que o processo cego ou duplamente cego possui críticas. A necessidade de mudanças no processo editorial alinha-se à própria evolução das ciências, que tem apresentado alterações em seus processos, com o uso cada vez maior, de tecnologia da informação e comunicação.

Por fim, esperam-se estudos mais aprofundados sobre o RAP, no Brasil, haja vista que a tradicional forma de avaliação fechada apresenta inúmeras críticas, mesmo sendo a mais aceita pela comunidade acadêmica. A implantação da avaliação aberta é um processo mundialmente irreversível, o qual permitirá, em futuro breve, o acompanhamento e a avaliação, culminando com procedimentos transparentes, que fornecerão novos subsídios, tanto para os editores, quanto para os avaliadores-avaliados.

\section{REFERÊNCIAS}

ALBAGLI, S.; CLINIO, A.; RAYCHTOCK, S. Ciência Aberta: correntes interpretativas e tipos de ação. Liinc em Revista, v. 10, n. 2, 2014. DOI: 10.18617/liinc.v10i2.749. Disponível em: http://revista.ibict.br/liinc/article/view/3593. Acesso em: 31 mar. 2020.

ALEXANDER, D. et al. Academic publishing in disaster risk reduction: past, present, and future. Disasters, 2020. DOI: .10.1111/disa.12432. Disponível em: https://onlinelibrary.wiley.com/doi/abs/10.1111/disa.12432. Acesso em: 31 mar. 2020.

AMARAL, J. C. do; PRÍNCIPE, Eloísa. Ciência aberta e revisão por pares: aspectos e desafios para a participação da comunidade em geral. Cadernos BAD, n. 1, p. 320-325, 2018. Disponível em: https://www.bad.pt/publicacoes/index.php/cadernos/article/view/1934/pdf. Acesso em: 31 mar. 2020 BANKS, G. C. et al. Answers to 18 questions about open science practices. Journal of Business and Psychology, v. 34, n. 3, p. 257-270, 2019. DOI: 10.1007/s10869-018-9547-8. Disponível em: http://link.springer.com/10.1007/s10869-018-9547-8. Acesso em: 31 mar. 2020.

BARRAVIERA, B. et al. O Alinhamento das revistas brasileiras sobre Medicina Tropical e Doenças infecciosas e Parasitárias às práticas da Ciência Aberta. In: SHINTAKU, M.; SALES, L. F; COSTA, M. (Org). Tópicos sobre dados abertos para editores científicos. Botucatu, SP: ABEC, 2020. p. 215-230. DOI: 10.21452/978-85-93910-04-3.cap17. Disponível em: https://www.abecbrasil.org.br/arquivos/Topicos_dados_abertos_editores_cientificos.pdf. Acesso em: 31 mar. 2020. 
BARRETT, S. C. H. Proceedings B 2018: the year in review. Proceedings of the Royal Society B: Biological Sciences, 2018. DOI: 10.1098/rspb.2018.2590. Disponível em: https://royalsocietypublishing.org/doi/10.1098/rspb.2018.2590. Acesso em: 31 mar. 2020. 2019. BARRETT, S. C.H. Proceedings B 2019:the year in review. Proceedings of the Royal Society B: Biological Sciences, 2019. DOI: 10.1098/rspb.2019.2626. Disponível em: https://royalsocietypublishing.org/doi/10.1098/rspb.2019.2626. Acesso em: 31 mar. 2020. BRAVO, G. et al. The effect of publishing peer review reports on referee behavior in five scholarly journals. Nature communications, v. 10, n. 1, p. 322, 2019. DOI: 10.1038/s41467-018-08250-2. Disponível em: http://www.nature.com/articles/s41467-018-08250-2. Acesso em: 31 mar. 2020. 2019. COLE, J. R. Balancing acts: Dilemmas of choice facing research universities. Daedalus, v. 122, n. 4, p. 1-36, 1993. Disponível em: https://www.jstor.org/stable/20027197?seq=1. Acesso em: 31 mar. 2020.

DAVID, P. A. Reputation and Agency in the Historical Emergence of the Institutions of "open Science". In: National Academy of Sciences Conference on Science, Technology and the Economy, 1991, Irvine, California. Proceedings [...]. Irvine: National Academy of Sciences , 1991. FALAGAS, M. E. Peer review in open access scientific journals. Open medicine, v. 1, n. 1, p. e49, 2007.

FORD, E. Moving peer review transparency from process to praxis. Insights, v. 32 , 2019. DOI: 10.1629/uksg.480. Disponível em: http://insights.uksg.org/articles/10.1629/uksg.480/. Acesso em: 31 mar. 2020.

GIL, A. C. Como elaborar projetos de pesquisa. São Paulo: Atlas, 2008.

GINSPARG, P. ArXiv, 20. Nature , v. 476, n. 7359, p. 145-147, 2011. DOI: 10.1038/476145a. Disponível em: http://www.nature.com/articles/476145a. Acesso em: 31 mar. 2020. 2019. HARNAD, S. et al. The Access/Impact Problem and the Green and Gold Roads to Open Access. Serials Review, v. 30, n. 4, p. 310-314, 2004. DOI: 10.1080/00987913.2004.10764930. Disponível em: http://www.tandfonline.com/doi/abs/10.1080/00987913.2004.10764930. Acesso em: 31 mar. 2020. HARZING, A.-W. Two new kids on the block: How do Crossref and Dimensions compare with Google Scholar, Microsoft Academic, Scopus and the Web of Science?.Scientometrics, v. 120, n. 1, p. 341-349, 2019. DOI: 10.1007/s11192-019-03114-y. Disponível em: http://link.springer.com/10.1007/s11192-019-03114-y. Acesso em: 31 mar. 2020.

JANA, S. A history and development of peer-review process. Annals of Library and Information Studies , v. 66, n. 4, p. 152-162, 2019. Disponível em: http://hdl.handle.net/10760/39332. Acesso em: 31 mar. 2020.

KLEBEL, T. et al. Peer review and preprint policies are unclear at most major journals.

Scientific Communication and Education, 2020. Disponível em:

http://biorxiv.org/lookup/doi/10.1101/2020.01.24.918995. Acesso em: 31 mar. 2020.

LOBO, M. P. 2019. Peer Reviewers as Co-authors. OSF Preprints. April 2020.

DOI: 10.31219/osf.io/jtnq2. Disponível em:

http://biorxiv.org/lookup/doi/10.1101/2020.01.24.918995. Acesso em: 31 mar. 2020.

MILHAM, M. P.; KLEIN, A. Be the change you seek in science. BMC biology, v. 17, n. 1, p. 27 , 2019. DOI:10.1186/s12915-019-0647-3. Disponível em:

https://bmcbiol.biomedcentral.com/articles/10.1186/s12915-019-0647-3. Acesso em: 31 mar. 2020. POLKA, J.; ROSS-HELLAUER, T.; MCDOWELL, G. Opening Up Peer-Review Policies. Science Editor, v. 42, n. 2, p. 44-46, 2019. Disponível em: https://www.csescienceeditor.org/article/openingup-peer-review-policies/. Acesso em: 31 mar. 2020.

RABKINA, N. Open Peer Review: features, benefits and considerations for scholarly communication. In: HOLLISTER, C. (org.). Perspectives on Scholarly Communication: volume 1. Volume 1. Buffalo; University at Buffalo, 2019. p. 85-94. Disponível em: https://ubir.buffalo.edu/xmlui/handle/10477/81267. Acesso em: 31 mar. 2020. 
RAUPP, F. M.; BEUREN, I. M. Metodologia da Pesquisa Aplicável às Ciências. In: BEUREN, I. M. (Org.). Como elaborar trabalhos monográficos em contabilidade: teoria e prática. São Paulo: Atlas, p. 76-97, 2013.

ROOYEN, S. van et al. Effect of open peer review on quality of reviews and on reviewers' recommendations: a randomised trial. BMJ, v. 318, n. 7175, p. 23-27, 1999. DOI: 10.1136/bmj.318.7175.23.

ROSS-HELLAUER, T. What Is Open Peer Review? A Systematic Review. F1000Research, v. 6, n. 588, 2017. DOI 10.12688/f1000research.11369.2. Disponível em: https://f1000research.com/articles/6-588/v2. Acesso em: 31 mar. 2020.

ROSS-HELLAUER, T.; GÖRÖGH, E. Guidelines for open peer review implementation. Research Integrity and Peer Review, v. 4, n. 1, p. 4, 2019. DOI: 10.1186/s41073-019-0063-9. Disponível em: https://researchintegrityjournal.biomedcentral.com/articles/10.1186/s41073-019-0063-9. Acesso em: 31 mar. 2020.

SCHMIDT, B. et al. Ten considerations for open peer review. F1000 Research, v. 7, 2018. DOI: 10.12688/f1000research.15334.1. Disponível em: https://f1000research.com/articles/7969/v1. Acesso em: 31 mar. 2020.

SILVA, J. A. T. da Challenges to open peer review. Online Information Review, v. 43, n. 2 , p. 197-200, 2019. DOI: 10.1108/OIR-04-2018-0139. Disponível em:

https://www.emerald.com/insight/content/doi/10.1108/OIR-04-2018-0139/full/html. Acesso em: 31 mar. 2020.

WALSH, E. et al. Open peer review: a randomised controlled trial. British Journal of Psychiatry, v. 176, n. 1, p. 47-51, 2000. DOI: 110.1192/bjp.176.1.47. Disponível em:

https://www.cambridge.org/core/product/identifier/S0007125000263757/type/journal_article.

Acesso em: 31 mar. 2020. 